\title{
REAPROPIACIÓN Y RESIGNIFICACIÓN DEL TERRITORIO Y EL PATRIMONIO: APLICACIÓN DE LA EVALUACIÓN DE ACOGIDA A LA ZONA DE DOS HUECOS, ARGENTINA
}

\author{
Guillermina Fernández ${ }^{1}$ \\ Susana Ricci ${ }^{2}$ \\ Silvia Valenzuela ${ }^{3}$ \\ Aldo Ramos ${ }^{4}$
}

Centro de investigaciones y estudios ambientales. Facultad de Ciencias Humanas. UNICEN. Tandil. Corresponding e-mail: aldogramos@gmail.com

(Fecha envío: 10/05/16 - Fecha aceptación: 19/06/16)

\section{Resumen}

La reapropiación del patrimonio territorial implica la legitimación social y el otorgamiento de un valor simbólico lo cual implica una resignificación del mismo por parte de la sociedad, produciendo como resultado un nuevo proyecto territorial. En algunos casos, como en el de estudio, se vincula la incorporación del mismo al sistema productivo como recurso turístico-recreativo. Por esto es necesario procesos de integración basados en tres aspectos básicos: la integración física, funcional y social de la propuesta.

El objetivo del trabajo es analizar el patrimonio natural y cultural del área denominada Dos Huecos (Parque Antonio y Juana Cinque de Tandil. Buenos Aires) y su potencial reutilización para fines recreativos.

La metodología aplicada es de tipo exploratoria-descriptiva, apoyada en el análisis de las características ambientales que conforman la estructura y el funcionamiento del territorio, mediante matrices de capacidad de acogida. Como resultado puede decirse que en términos potenciales es viable la reutilización del Parque Antonio y Juana Cinque, como mecanismo de reapropiación del patrimonio local, bajo ciertas condiciones e integrando el área al contexto espacial y temporal que le otorga significado.

\section{Palabras claves}

resignificación, reapropiación, patrimonio natural, patrimonio cultural, evaluación, territorio

\section{Abstract}

The reappropriation of territorial heritage involves social legitimacy and providing a symbolic value which implies a redefinition of it by society, producing as a result a new territorial project. In some cases, as in the study, these processes involve the incorporation of it into the production system as a tourist-recreational resource. For this you need integration processes based on three basic aspects: physical, functional and social integration of the proposal.

The aim of this work is to analyze the natural and cultural heritage of the area called Two Hollows (Antonio and Juana Cinque Tandil Park. Buenos Aires) and its potential reuse for recreational purposes.

The methodology is exploratory and descriptive, based on the analysis of the environmental characteristics that make up the structure and functioning of the territory by matrices of carrying capacity type. As a result, it can be said that in terms is viable potential reuse of Antonio and Juana Cinque Park as a mechanism of reappropriation of local heritage, under certain conditions and integrating the area to the spatial and temporal context that gives it meaning.

\section{Keywords}

resignification, appropriation, natural heritage, cultural heritage, assessment, territory

\footnotetext{
${ }^{1}$ Profesora y Licenciada en Geografía. Master en gestión pública del turismo.

${ }^{2}$ Licenciada en ecología. Doctora en Ciencias Naturales.

${ }^{3}$ Profesora de Geografía. Master en evaluación de impacto ambiental.

${ }^{4}$ Profesor y Licenciado en Geografía. Master en ecoauditorias y planificación empresarial del medio ambiente.
} 


\section{INTRODUCCIÓN}

Los procesos de reapropiación del patrimonio suponen la legitimación de la sociedad y el otorgamiento en el presente de un valor simbólico a un bien, proceso o rasgo cultural o natural. Esta resignificación adquiere múltiples facetas y es un proceso incentivado por las condiciones y valores de la sociedad actual.

Diversas formas de apropiación a lo largo del tiempo y como resultado de representaciones y acciones diferentes, dan como resultado múltiples proyectos territoriales asociados a la reutilización del patrimonio. Algunos suponen la incorporación de este a un sistema productivo asignándole un carácter específico (por ejemplo, recurso turístico-recreativo) que le otorgan un valor de uso. La viabilidad y sustentabilidad del proyecto radica en los actores, los motivos de uso y la gestión y las tensiones que puedan generarse en el campo social.

La importancia de la apropiación de los actores sociales del patrimonio y de sus elementos a escala territorial local es clave para entender los procesos de transformación de dichos espacios y de las lógicas de intervención. Pero como no todos los actores territoriales se vinculan de la misma manera a estos espacios o los valorizan de igual modo, los elementos en él constituidos, a partir de los intereses económicos o no que estos despierten, tejen un campo de fuerzas que requiere ser analizado para comprenderlos y proyectar los usos que de ellos se desprendan.

Se requieren procesos de integración basados en tres aspectos fundamentales: la integración física, funcional y social de la propuesta. El tratamiento de reapropiación a escala territorial exige un trabajo previo de planificación territorial que asegure el uso correcto y efectivo del patrimonio como elemento de desarrollo social y económico. Teniendo en cuenta lo expresado, el objetivo es analizar el patrimonio natural y cultural del área denominada Dos Huecos (Tandil, Argentina) y su potencial reutilización para fines recreativos a partir de la integración de los aspectos mencionados. Para esto se aplicará la metodología de evaluación de la capacidad de acogida del territorio.

\section{RESIGNIFICACIÓN Y REAPROPIACIÓN: TERRITORIO Y PATRIMONIO}

Existen diferentes formas de analizar el presente de un territorio en permanente transformación y de dar tratamiento al patrimonio en él contenido. En primer término, podemos aproximarnos a los debates en torno a los conceptos de resignificar y reapropiar y su vínculo con el patrimonio y con el territorio. Respecto al primero, no existe definición, aunque pertenece a un amplio campo de aplicación; podemos partir de su vinculación con la idea de otorgar nuevos significados a conceptos o a perspectivas ya dadas. Como sostiene Molina Valencia (2013:3) "una premisa fundamental es que la acción se basa en significados que son adquiridos socialmente en la interacción, en contextos particulares, y por consiguiente marcos simbólicos diferenciales. La amalgama de todas las interacciones, formas y contenidos define de forma singular la subjetividad y la identidad colectiva expresadas en acciones, constituyendo así aquello que sería susceptible de ser transformado, resignificado". Considerando esto, la resignificación del territorio implicaría la intencionalidad de un grupo social de dotarlo de nuevas acepciones, producto del devenir histórico o de la propia lectura que la sociedad hace de un determinado espacio.

En relación al concepto de reapropiación se puede partir de considerar el significado que el diccionario da al termino apropiar: "Hacer propia de alguien una cosa". Y si se suma el prefijo re este sugiere "repetición o nueva acción de algo". Entonces la reapropiación implicaría una nueva forma por la cual un sujeto o grupo social se apropia de una cosa o bien, que no le es del todo suyo. Por ende y en relación al patrimonio, puede incluir a los procesos de acercamiento del patrimonio a grupos que no tienen con él un vínculo directo (temporal o espacial).

Con otra perspectiva, desde la economía ecológica, la apropiación de la naturaleza ha sido tratada por Enrique Leff (2005), como una estrategia de poder, que moviliza a la sociedad dándole fuerza política; y en esta línea el movimiento se expresa, según el autor, no solo como una lucha por mejorar la calidad de vida, sino que conduce a un proceso de reapropiación de la naturaleza por la sociedad reduciendo la parte de la naturaleza que podría ser apropiada por el capital e implicando procesos de autonomía y autogestión de la población en relación al manejo sustentable de su ambiente. El territorio es un espacio donde se precipitan tiempos diferenciados, donde se articulan identidades culturales y potencialidades ecológicas. Es pues el lugar de convergencia de los tiempos de la sustentabilidad: los procesos de restauración y productividad ecológica; de innovación y asimilación tecnológica; de reconstrucción de identidades culturales... El territorio es el locus de las demandas y los reclamos de la gente para reconstruir sus mundos de vida. El nivel local es donde se forjan las identidades culturales, donde se expresan como una valorización social de los recursos económicos y como estrategias para la reapropiación de la naturaleza"

Entonces, se está en presencia de dos procesos vinculados a la propia construcción social del territorio como patrimonio o como continente de un patrimonio, que también es otra construcción social de carácter simbólico. Entonces, los procesos de patrimonialización, de apropiación o reapropiación del mismo serán complejos, puesto que involucran la percepción y significación que diferentes grupos sociales hacen de él; lo que resulta en valores diferentes, vínculos distintos y lógicas de intervención no siempre homogéneas, ni compatibles. 
Ser patrimonializable es una cualidad de un elemento tangible o intangible o de un territorio como legado, y reside en su capacidad de ser reapropiado y convertirse en referente simbólico. Esta situación es cambiante, puesto que a lo largo del tiempo la sociedad otorga significatividad a diferentes manifestaciones o elementos, y en ese devenir, va seleccionando y descartando bienes tangibles e intangibles, que son destacados. Otros persisten o desaparecen, y solo los que quedan como relictos, pueden ser resignificados en procesos posteriores.

El territorio analizado como patrimonio es resignificado, reapropiado, como mecanismo de valorización actual y legado. Entonces se parte de la noción de patrimonio como algo que está en constante interacción con las culturas del presente, que es activado en un único tiempo que incluye su relación con el pasado y su proyección al futuro. El valor del patrimonio responde a su autenticidad, pero sobre todo a su reconocimiento: puesto que se trata de algo en lo que una colectividad concreta se reconoce a sí misma y como parte de su historia y vida cultural. En este sentido Lowenthal (1998:18) expresa que la condición de legado o herencia queda mediada por los procesos de selección y activación, a partir de lecturas e interpretaciones específicas y en función de necesidades e intenciones actuales. Cada generación determina su propio legado eligiendo lo que quiere descartar, ignorar, tolerar o atesorar y la forma de tratar lo que está guardado. Y esto media en ambos procesos en todo el territorio, o parches de este, en aquellas áreas que parecen no tener uso actual, o han perdido sentido, o presentan relictos de procesos de intervención ya obsoletos o desaparecidos.

Al respecto, siguiendo a Thompson (Thompson, 1993 en Valdebenito) se podrían distinguir dos grandes procesos de valoración social del patrimonio cultural. Una valoración simbólica, en virtud de la cual estos bienes adquieren forma en la medida en que son estimados por los individuos que los producen y reciben. Pero también la activación patrimonial puede tener como motivación una valoración económica, es decir ser concebidos como bienes que podrían ser intercambiados en un mercado, adquiriendo con ello la categoría de formas simbólicas mercantilizadas. La producción del valor y apropiación del patrimonio cultural como manifestaciones compartidas y vividas de una cultura, se vinculan directamente a las especificidades del contexto socio-territorial del cual estos son símbolos característicos. Territorializar el patrimonio, entonces, significa destacar que éste es expresión de una comunidad particular, en el sentido que se encuentra siempre arraigado a un espacio propio. Lo que implica entender el territorio no sólo como un espacio sobre el cual transcurre la vida social, sino un artífice de esa realidad, un territorio históricamente construido en el cual ocurren y se han desarrollado determinados procesos sociales y culturales que intervienen en la experiencia de vida de sus habitantes.

Por tal razón la escala local es la contextualización, ese locus donde ocurre la dinámica que implica la relación de la sociedad con la naturaleza y con las sucesivas transformaciones que de esta relación devengan; esta es la clave. Esto se presenta como un campo tensional basado en la relación que los distintos grupos sociales tienen con ese espacio producto de procesos simbólicos y afectivos que permiten la construcción de lazos y sentimientos de pertenencia. Pero también de las coyunturas contextuales de esos grupos y de intereses en el corto, mediano y largo plazo. Desde ese punto se transforma el territorio, no siempre mediado por procesos conscientes de activación o destrucción del patrimonio, pero requiere a posteriori analizar cómo plasmar eso en proyectos concretos que requieren examinar las capacidades reales que tiene para ser reactivado en función de lógicas de intervención que sean las más adecuadas.

A partir de estos ejemplos la pregunta que debe plantearse es ¿Cómo se reapropian o resignifican estos territorios que hoy son disfuncionales, o aquellas partes que están abandonadas o degradadas y que son manifestaciones potenciales de un patrimonio local? En general estos sitios contienen muchos elementos que pueden ser considerados como formas simbólicas o soporte de significados culturales para determinados grupos. Contiene los modos de comportamiento, las prácticas sociales, los usos y costumbres, los procesos productivos, la organización del espacio y del tiempo, entretejidos y evidenciados en relictos de construcciones, de maquinarias, en relatos, objetos, etc. y en el propio paisaje actual.

Así la recreación se convierte en un potencial reactivador de esos espacios, porque permite la resignificación (tanto de aquellos que disfrutan las áreas como de quienes las gestionan o de los que se vinculan indirectamente) y la apropiación de una variedad de actores. Pero también genera transformaciones y dinámicas que es necesario evaluar para evitar que sólo se resignifique bajo los intereses de algunos en detrimento de otros, o solo sea apropiada por sectores, excluyendo al resto de la comunidad, o grupos que forman la trama territorial compleja.

Por lo tanto, una vez comprendidos los alcances de los procesos de reapropiación del patrimonio y del territorio, es necesario pensar las actividades o usos que permiten resaltar esos elementos, manteniendo ese valor simbólico aún en la búsqueda de rentas económicas producto del usufructo del mismo.

La gestión integrada del territorio se debe centrar en plasmar estrategias de planificación y gestión de los recursos, el patrimonio y el espacio, interconectando a la trama social, económica y ecológica los elementos y los usos. Dichos procesos deben llevarse a cabo en una primera etapa a partir del análisis espacial y el diagnóstico de los bienes, rasgos o procesos que hacen a la identidad y por ende son manifestaciones territoriales patrimonializables. Posteriormente es necesario establecer la relación actual de ese territorio con los grupos 
sociales que se identifican con él, lo usan, le dan valor y lo resignifican cotidianamente, aunque no sea siempre visible.

El carácter patrimonial del territorio se manifestaría entonces en dos ámbitos diferentes; en su dimensión ambiental, representada por los recursos y espacios naturales de interés y en su dimensión histórica y social que está presente en la organización y conformación espacial, urbana y arquitectónica del mismo, de la cual existen huellas identificables en el territorio que poseen el valor de reflejar el modo de ser y habitar de una sociedad determinada, o de varias sociedades a lo largo del tiempo.

El patrimonio se contempla como herencia, como legado, pero también como capacidad de actuación sobre el presente y el futuro a través del aprovechamiento de sinergias, de recursos humanos, ambientales, financieros, territoriales, etc. Es decir, se concibe el patrimonio en un sentido amplio que integra lo cultural, lo físicoambiental, lo natural, la dimensión económica y el componente espacial y territorial. Y, a su vez, se amplían las funciones que se le atribuyen al territorio: «El territorio (...) es la base idónea en la que entender el patrimonio y, además, en la que conectarlo con los grandes temas de interés del final del milenio: la preocupación medioambiental, el desarrollo sostenible, las nuevas tendencias demográficas, la distribución de las rentas, la sociedad de la comunicación, etc.» (IAPH: 9)

Como sostiene Yañez (2008:262), "uno de los argumentos más sólidos sobre el que descansa la valoración territorial del patrimonio reside en que existen muchos territorios con escasa presencia de bienes patrimoniales declarados que, sin embargo, si poseen otros recursos territoriales potenciales, entre ellos bienes culturales, que, precisamente por no poseer esa excepcionalidad y singularidad que justifica su declaración formal, pueden ser más fácilmente manipulados a través de planes de ordenación y puesta en valor que persigan unos fines de desarrollo determinados. Puesto que el patrimonio es mucho más amplio y numeroso que el patrimonio formalmente declarado y protegido, hay que encontrar nuevos espacios y metodologías, como las derivadas de la ordenación territorial, el planeamiento estratégico y las nuevas figuras de gestión, que permitan poner en valor estos recursos potenciales relacionándolos entre sí y sumando, por así decirlo, sus valores".

Para ello es necesario determinar la capacidad de esos recursos potenciales para acoger determinadas funcionalidades o usos que el territorio tiene para su reactivación.

En este contexto nace la planificación de los usos a partir de la evaluación de las aptitudes de determinado espacio y de los impactos que el destino o el uso propuesto tiene en él. Esto requiere el conocimiento del funcionamiento del sistema, en su complejidad y del patrimonio cultural y natural a él asociado, incluso en términos de integración paisajística.

Indudablemente estamos frente a un juego de escenarios que permiten reutilizar un determinado espacio. El mismo puede estar ya en proceso de reapropiación por diversos grupos sociales y a partir de allí se puede proponer su activación o readecuación. Por lo tanto, la evaluación territorial es siempre compleja, por la heterogeneidad de los elementos, su interdependencia y la permanente necesidad de encontrar ligazones entre ellos y relaciones con otros sistemas por fuera.

El conocimiento del territorio y su evaluación en función de nuevos usos es esencial en los procesos de planificación asociados a diferentes formas de apropiación del patrimonio. La evaluación basada en el concepto de que todo territorio tiene una capacidad para acoger nuevos usos, plantea en términos teóricos el uso óptimo, que se apoya sobre dos pilares: el análisis de aptitudes y de impactos.

Es una de las posibilidades que pretende explorar este trabajo, el valor y resignificado que las sierras de Tandil tienen para los residentes y para los turistas supone procesos de apropiación, incluso de áreas aparentemente degradadas, que requieren ser analizadas, para conocer cuál es la capacidad de esos territorios para dar respuesta a esa necesidad del presente de articulación territorial.

\section{METODOLOGÍA}

La propuesta metodológica aplicada denominada Evaluación de la Capacidad de Acogida de un Territorio (ECAT), nace dentro de las teorías sobre planificación física del territorio, con una fuerte impronta ecológica y actualmente ha dado paso a la incorporación de una valoración social del mismo para el análisis de las aptitudes y los impactos.

En los procesos de reapropiación del patrimonio, éste posee intrínsecamente una aptitud que lo potencia y limita, estableciendo su vulnerabilidad, su fragilidad o debilidad, sus fortalezas y rasgos sobresalientes. Desde esta "composición" y producto de la interrelación interna y externa entre elementos y sistemas, responderá frente al nuevo rol otorgado o uso propuesto; lo que podrá generar mayores o menores impactos.

La aptitud es definida como una valoración de oportunidades que el territorio ofrece y se relaciona con la fragilidad del mismo. Como resultado, pueden establecerse limitaciones o tipologías de intervención más adecuadas. Sin duda, se está en presencia de un modelo territorial ideal que requiere de un "arreglo a valores" 
de la sociedad con la que se vincula. Por lo tanto, se debe combinar con factores extrínsecos al área y contextuales al territorio, como son la accesibilidad y la viabilidad económica, política y social.

La ECAT es definida puede ser definida como "el grado de idoneidad o cabida que presenta un territorio para una actividad, teniendo en cuenta a la vez, la medida en que dicho medio cubre sus requisitos y los efectos que la misma pueda generar" (Gómez Orea, 1992:25). Es una relación de reciprocidad entre ambos factores donde, producto de un proceso de reapropiación de un área (espacios mineros abandonados en este caso), se establece el grado de incidencia que las actividades asociadas a este puedan generar tanto de tipo positivas como negativas.

Dentro de los mecanismos para llevar a cabo esta propuesta analítica es necesario determinar dos posibles caminos para la interpretación y análisis de un área. Esto refiere a como se estructura el espacio a evaluar: como un continuo de caracteres cambiantes o como una estructura discontinua de diferentes unidades relativamente homogéneas. En el primer caso, es reconocer todos los factores y elementos que caracterizan el territorio y evaluarlos particularmente para luego relacionarlos. Así se llevan a cabo inventarios que luego son seleccionados y valorados a partir de un objetivo concreto. Son muy comunes en el tratamiento del patrimonio, puesto que se enfoca particularmente en él y no tanto en el entorno.

La segunda vía es la de tipo sintética, partiendo de la delimitación previa de unidades con caracteres homogéneos en forma integrada. En la mayoría de los casos se delimita en función de su apariencia o aspecto externo (Ej.: unidades de paisaje), o por procesos de identificación perceptiva de la sociedad (Ej.: la zona del cerro, la zona del arroyo, la zona de la Cruz). En este caso, los procesos evaluativos se dan para toda la unidad y no factor por factor. Este tipo de procedimientos está comúnmente vinculado al análisis multicriterio, que permite combinar la preferencia o selección (la valoración) entre un conjunto de alternativas reales, en presencia de criterios diversos (tema que no se desarrolla en esta presentación).

De esta manera la metodología aplicada es de tipo exploratoria-descriptiva, apoyada en técnicas de observación directa y entrevistas a informantes calificados (Asociación de Guías de Turismo de Tandil).

A partir del análisis de las diferentes características ambientales que conforman la estructura y funcionamiento del territorio, se elaboró la síntesis. La información de las unidades se combinó con las diferentes actividades recreativas potenciales, generando matrices de aptitud, de impacto y por último, la matriz de capacidad de acogida.

La metodología empleada parte de la síntesis territorial a través de:

- La definición de unidades síntesis territoriales: a partir de rasgos particulares dentro del área (ecológicos, productivos, paisajísticos, de protección)

- La construcción de la matriz de síntesis territorial: que relaciona las unidades identificadas con la relación que tienen respecto a rasgos ecológicos sobresalientes, rasgos patrimoniales, producción/nivel de uso, protección, estado de conservación y paisaje. Ponderados de 1 a 3 en función de las características que las definen.

- Definición de actividades: se establece un listado y caracterización de las mismas, teniendo en cuenta los usos que se determinan potenciales, definidos previamente, a partir de la relación que la sociedad actual tiene con el espacio y de los posibles mecanismos de apropiación.

- Construcción de la matriz de aptitud: donde se cruzarán las unidades de síntesis territorial con las diferentes actividades o usos asignados, resaltando qué actividades son más favorables a cada unidad (valorada de 0 a 3 , donde 0 supone no aplicable).

- La construcción de una matriz de impacto: donde se cruza la información de la unidad síntesis territorial con las actividades asignadas hipotéticamente. La valoración asignada estará en relación al impacto negativo que pueda generar y va de 0 a 3 , donde se parte de considerar una actividad favorable (0) a una de impacto alto.

- La construcción de la matriz de Capacidad de Acogida: resulta de la combinación de las dos matrices anteriores, donde se establece un valor síntesis que determina una clasificación empleada en la construcción de la matriz es:

Código 4: vocacional, la clase a que se asigna indica que es muy apta para acoger la actuación, tanto desde el punto de vista del uso como del conservacionista.

Código 3: compatible sin limitaciones, la clase a que se asigna, sin ser vocacionalmente adecuada para acoger la actuación, resulta aceptable desde los dos puntos de vista anteriormente mencionada. Puede sugerirse igual la necesidad de aplicar Evaluaciones de Impacto (EI)

Código 2: Compatible con limitaciones: se aplica a situaciones similares al caso anterior, pero con la condición de un control riguroso sobre la actuación en términos de diseño, tecnologías materiales, etc.

Código 1: incompatible, significa que la clase no reúne condiciones para la localización de la actividad desde el punto de vista del uso, de la conservación o de ambos.

Código cero: no aplicable, no tiene sentido la aplicación de la actividad en la clase a la que se somete. 


\section{4. ÁREA DE ESTUDIO}

La ciudad de Tandil se localiza en el sureste de la Provincia de Buenos Aires. Se encuentra a $350 \mathrm{Km}$. de la ciudad de Buenos Aires (Argentina) y la población actual es de 123.000 habitantes (Censo Nacional de Población y Vivienda 2010).

Mapa 1: Localización de Tandil en Argentina Fuente: elaboración personal

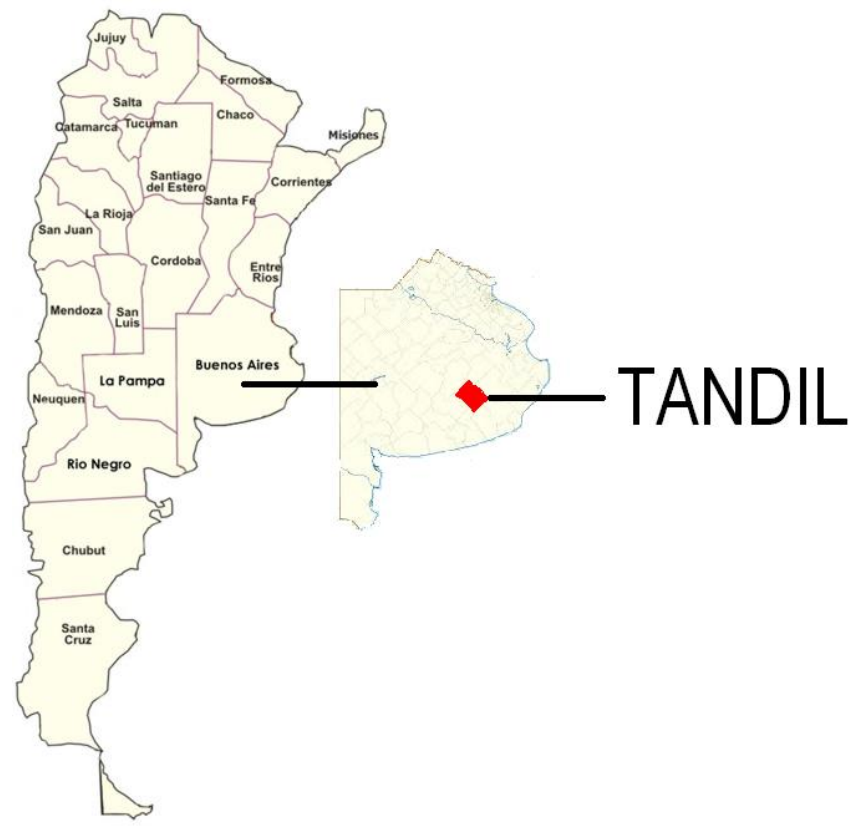

El Partido de Tandil está emplazado en la pampa húmeda y más precisamente, en el sistema serrano de Tandilia, que lo atraviesa en sentido NO-SE.

Este sistema orográfico se halla caracterizado por un conjunto de serranías bajas y de formas redondeadas en el centro y norte del partido, mientras que cambian a formas de mesas o tabulares hacia el oeste y sur. Con respecto al emplazamiento, la ciudad se extiende de forma irregular, en una superficie aproximada de $50 \mathrm{~km} 2 \mathrm{y}$ cuyo soporte se caracteriza por ser una encrucijada de valles que descienden desde las Sierras de Tandil hacia la llanura norte, continuándose hasta la depresión del río Salado. La ciudad de Tandil está en una zona de interfluvio formada por una lomada al pie y al norte del Cerro Independencia y cuya altura es relativamente baja (286 msnm).

Desde el punto de vista geológico el basamento cristalino precámbrico es expuesto principalmente en la zona central y septentrional del sistema, formando la mayor parte de las sierras de Tandil y Azul. Se trata de un complejo ígneo-metamórfico que forma parte del cratón del Río de la Plata, que se extiende desde el suroeste de Uruguay, a través de la isla Martín García, hasta las sierras de Tandilia. Este complejo ígneo-metamórfico o Complejo Buenos Aires constituye el basamento local al que pertenecen las sierras de la zona de Tandil, con alturas máximas de $300 \mathrm{msnm}$. Ubicada en el centro del sistema de Tandilia, el conjunto de serranías próximas a Tandil constituye el afloramiento de mayor antigüedad, entre 2.200 y 1.800 millones de años (Teruggi y Kilmurray, 1980: 921).

Estas características geológicas fueron las que permitieron que la explotación minera en Tandil haya sido una de las primeras actividades económicas desarrollada. La mayoría de ellas fueron localizándose principalmente en el área periurbana y rural, con una impronta que transformó el territorio de forma continua desde fines del siglo XIX hasta la actualidad, siendo relegada a zonas cada vez más periféricas, producto del surgimiento de un movimiento social ambientalista que tiene como objetivo la eliminación de actividades mineras y constructivas en el sector serrano más o menos próximo a la ciudad.

Dentro de la ciudad de Tandil, el área específica de estudio tiene una superficie de 40 hectáreas, ubicándose en los faldeos serranos del sector sur de la ciudad a unos $240 \mathrm{msnm}$ y se identifica catastralmente como Chacra 158, aunque su nombre por ordenanza municipal es "Parque Antonio y Juana Cinque". 


\section{Figura № 1: Área de estudio}

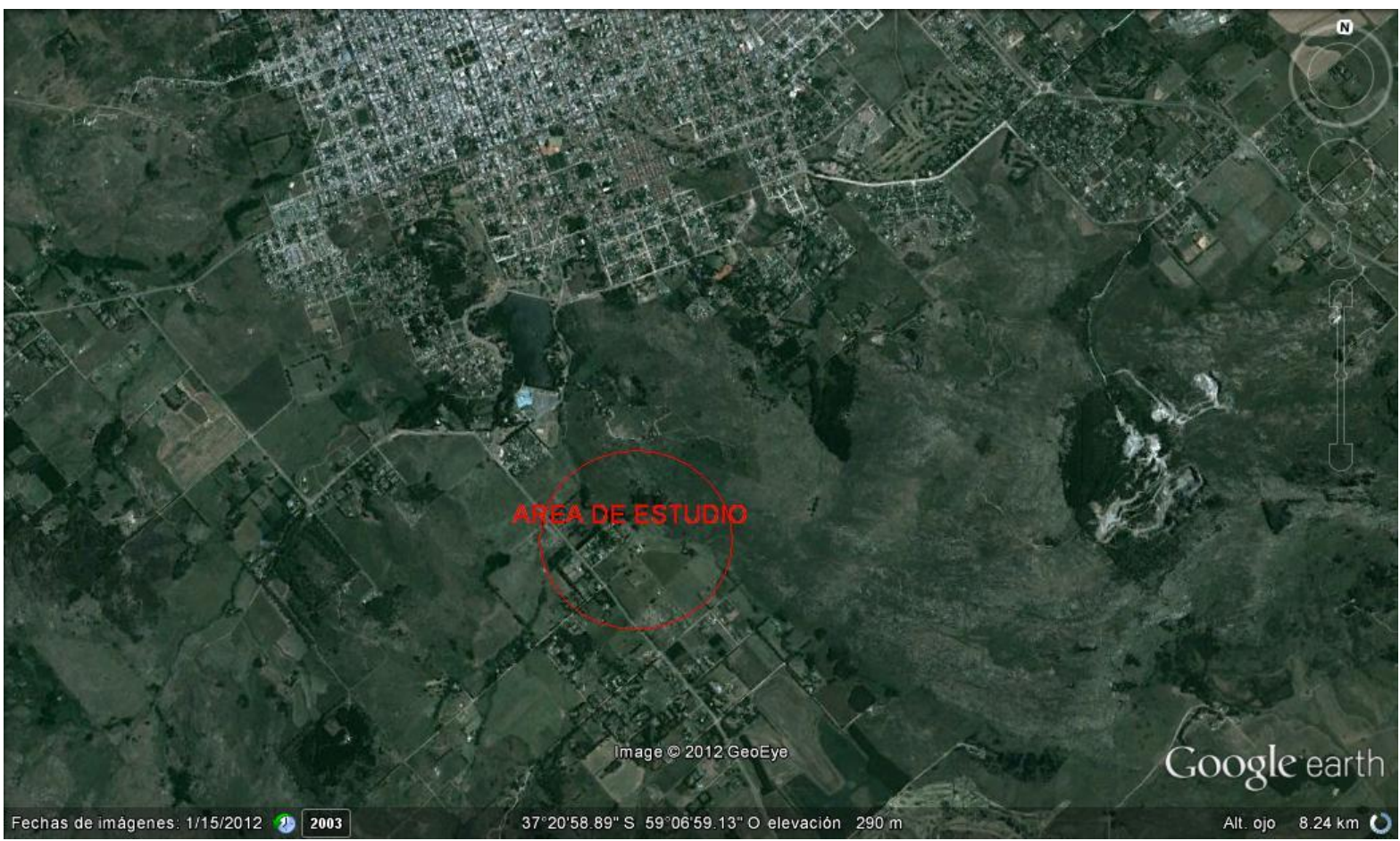

En la actualidad es de dominio municipal a partir de una donación de una familia de la comunidad que fuera oficializada por la Ordenanza Municipal N 8176 en el año 2000 y cuyo destino fuera el uso público y recreativo, según consta en el artículo 2 de la misma: ... aféctese el fundo como espacio verde público para la creación de un parque temático, histórico, cultural y recreativo para la ciudad de Tandil...

Toda el área está contemplada dentro de la Ley Provincial de Paisaje Protegido $N^{\circ} 14126 / 10$ y regulada por el Plan de Desarrollo Territorial de Tandil, del año 2005, en el cual la zona aparece como Zona de Servicios Extraurbanos (ZSE), es decir un área de potencial expansión residencial o de servicios asociados, hecho que marca una incongruencia con los usos públicos-recreativos que por ordenanza debe tener este sitio.

\section{Figura № 2: Delimitación Parque Antonio y Juana Cinque}

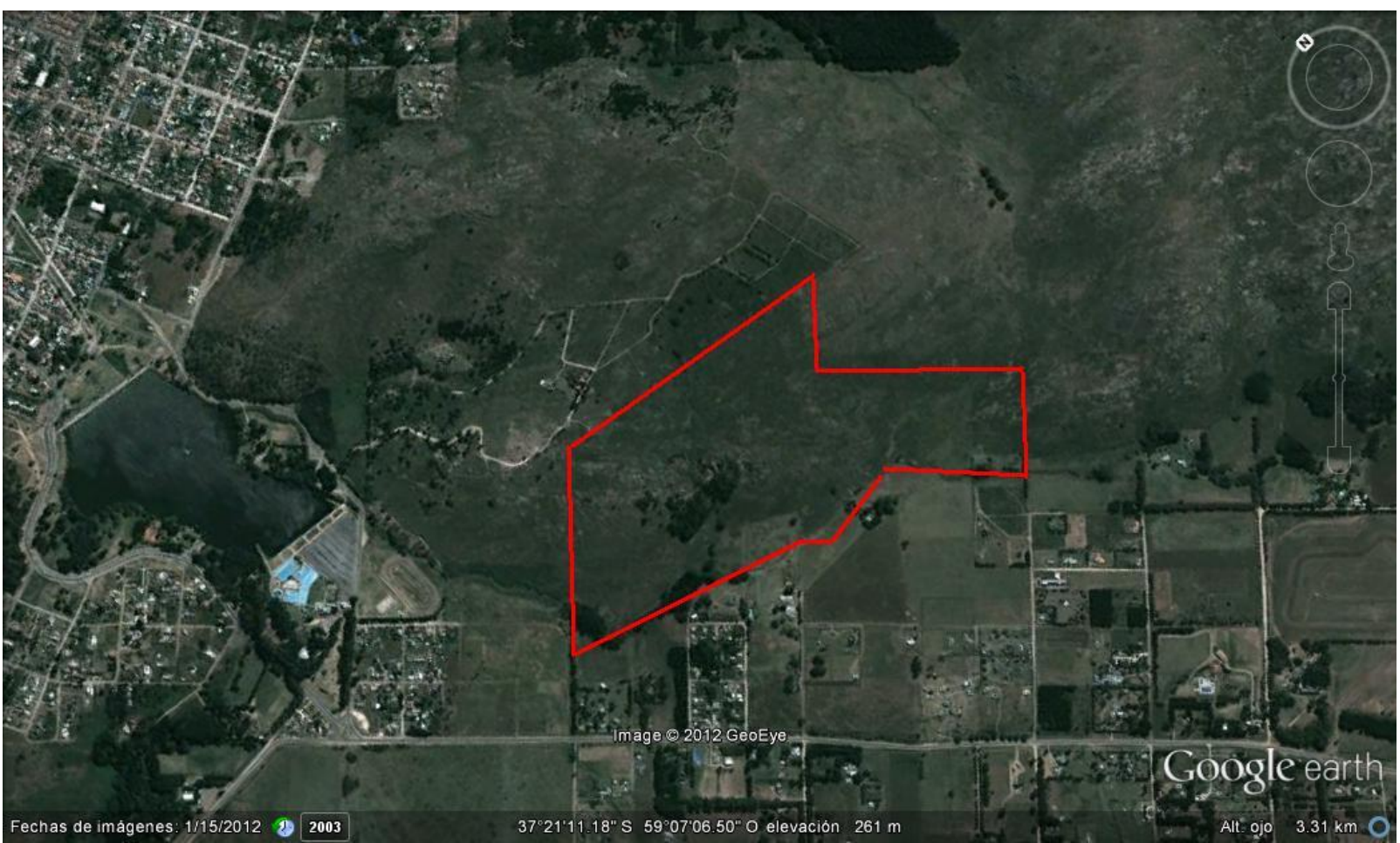


Fotos del área de estudio:

Foto 1: Frente de cantera

Fuente: archivo personal

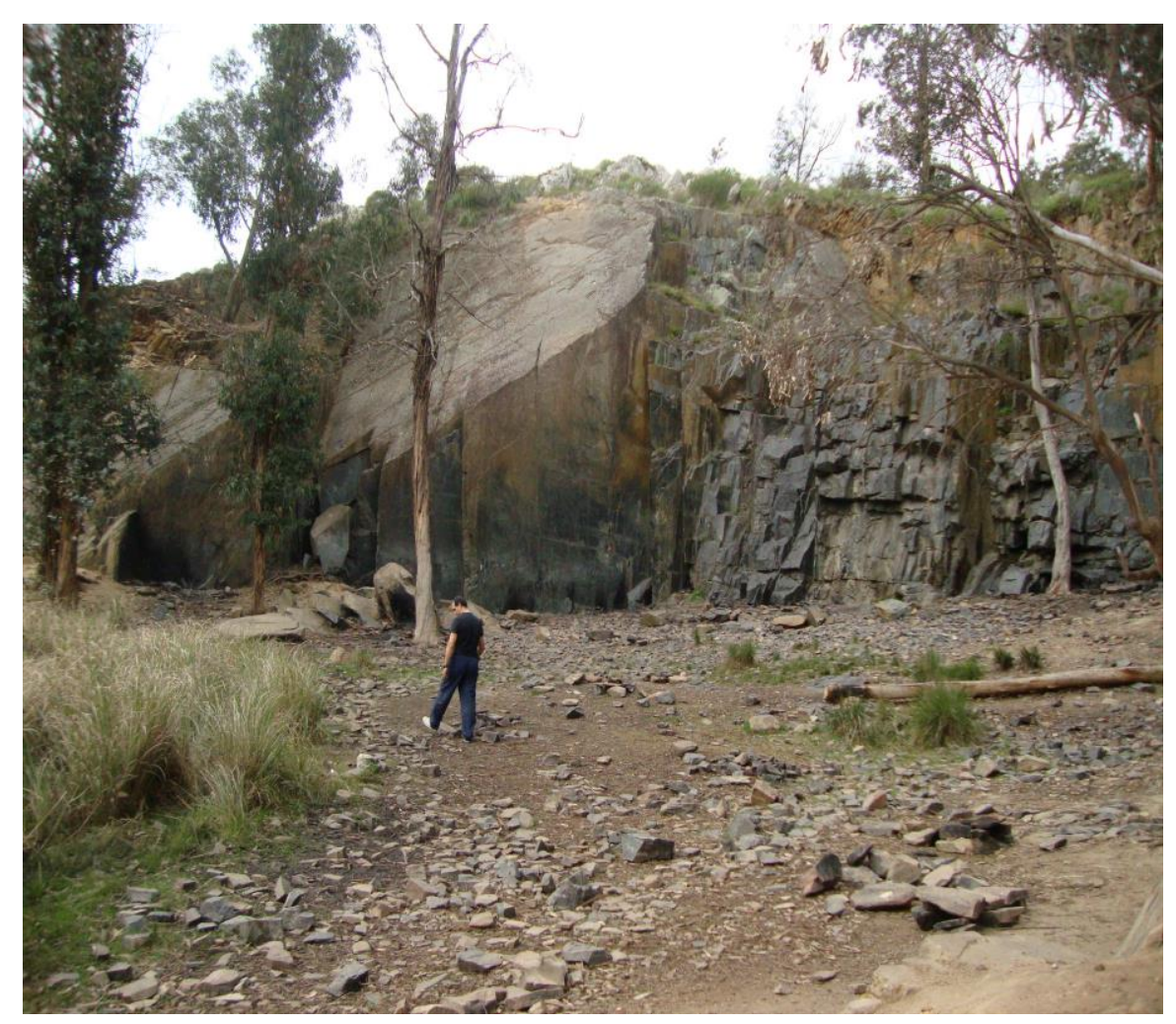

Foto 2: Frente de cantera, vegetación y actividades recreativas Fuente: Archivo personal

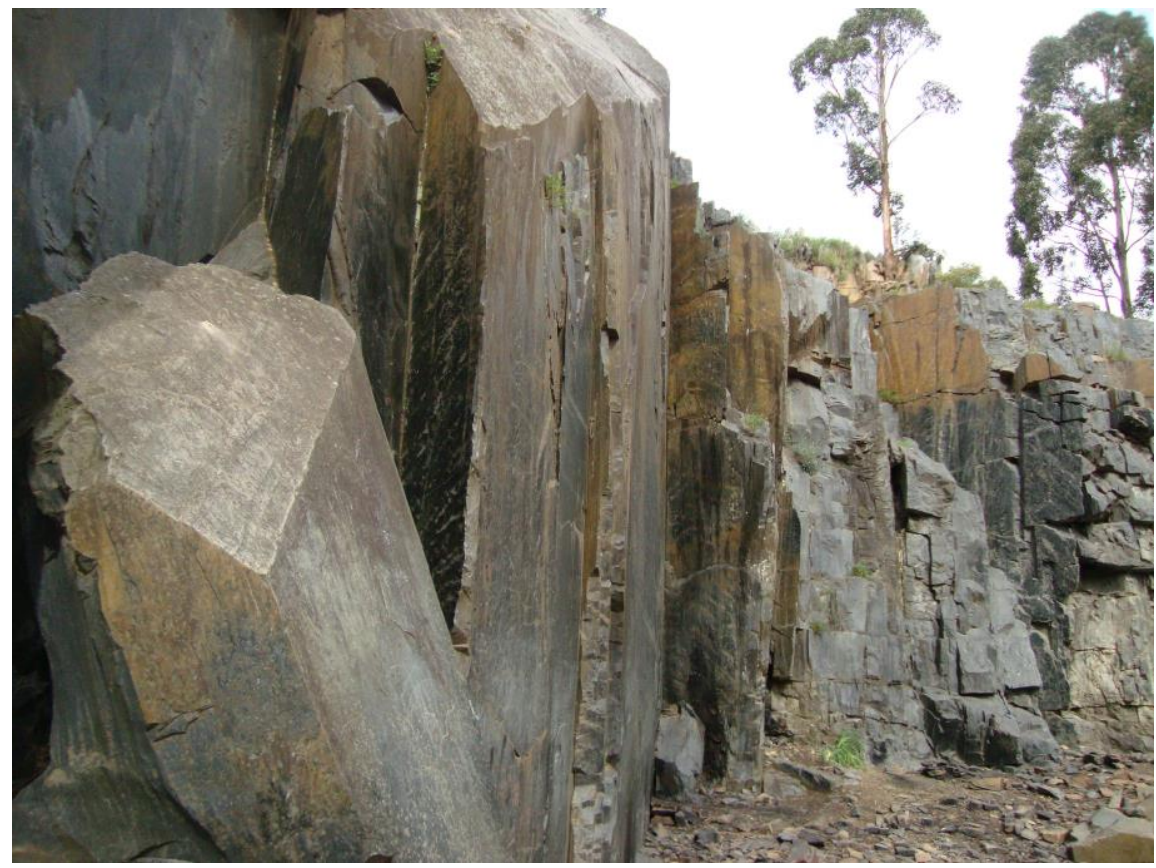


Foto 3: Actividades de rappel en frente de cantera Fuente: Archivo personal

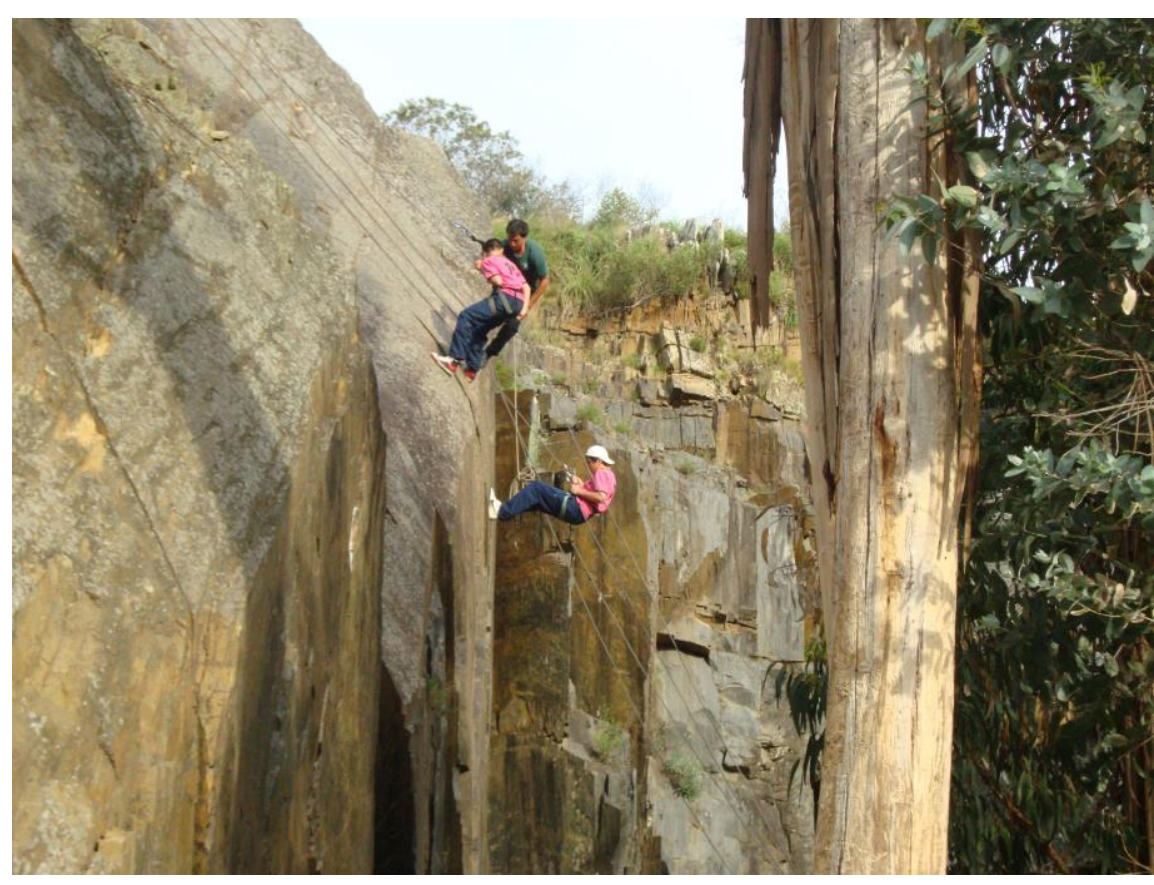

Foto 4: Vegetación colonizando área disturbada Fuente: Archivo personal

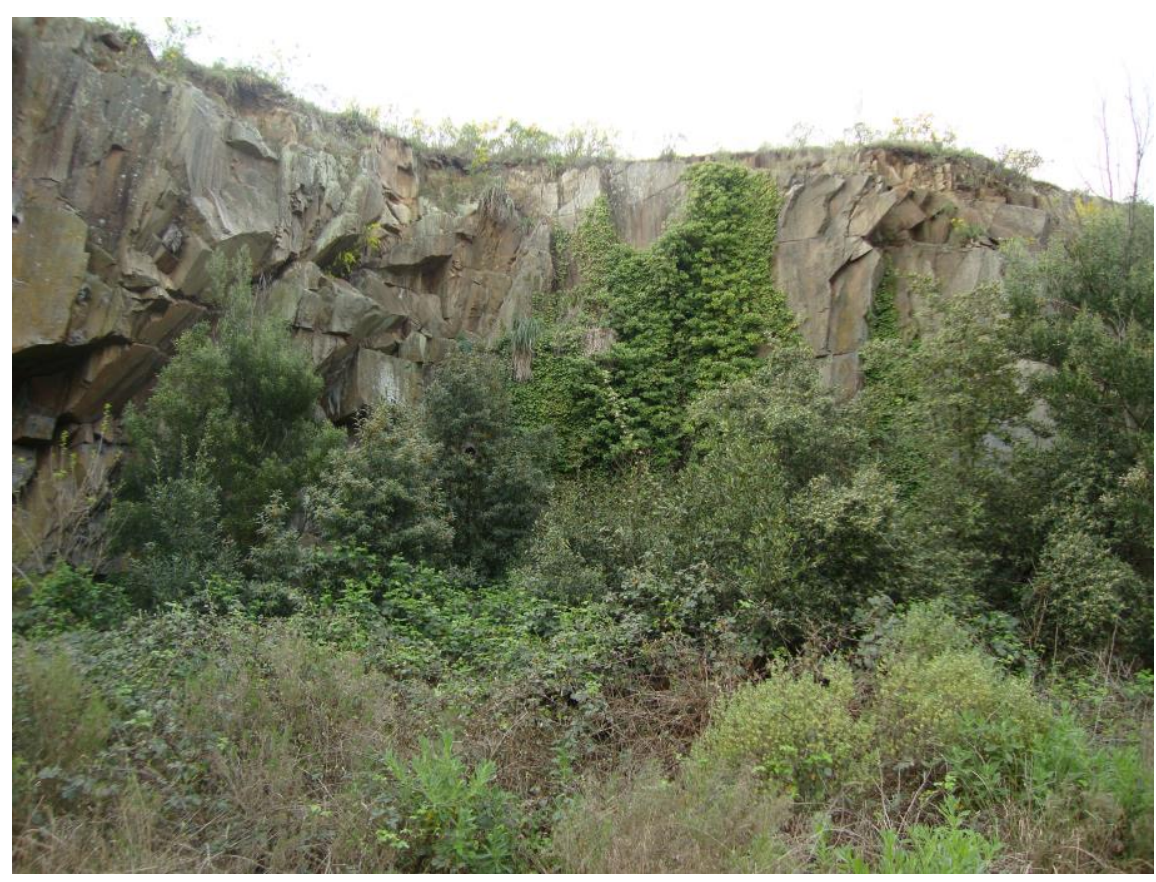


Foto5: Vistas del sector serrano Fuente: Archivo personal

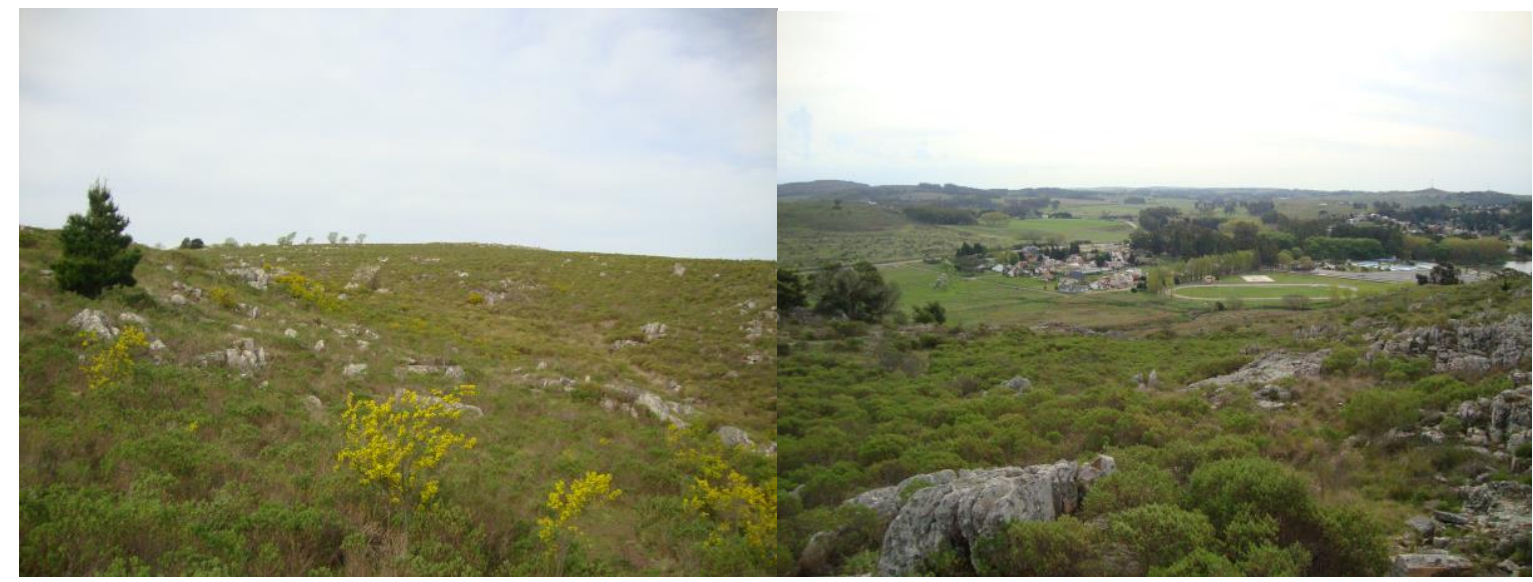

Se observa, en esta zona, una transición entre el sector urbano y el periurbano predominante y una pequeña porción que se comporta como rural. La misma está vinculada a la actividad recreativa del Dique Lago del Fuerte y al Mirador Cervantes, así como senderos espontáneos para actividades de aventura y deporte.

El uso residencial ha tenido un importante avance de la construcción sobre el sector serrano. En ella se encuentran dos paredes (pertenecientes a pequeñas áreas de explotación) conocidas como Dos Huecos. El sector más lejano al centro se convierte en un periurbano, con convivencia de usos residenciales y agrícolaganaderos.

\section{RESULTADOS}

A partir de la aplicación de la metodología ECAT se pudieran establecer tres unidades ambientales síntesis:

a. Área Dos Huecos (A1): que comprende el sector de canteras de granito inactivas con dos frentes de extracción y presencia de montes artificiales de eucaliptus, aromos y retamas. El uso es espontáneo de tipo recreativo, con actividades planificadas asociadas a turismo aventura.

b. Área de escurrimiento del arroyo Del Fuerte (A2): de curso permanente, escurre sus aguas hacia el Dique del Fuerte, de escasa profundidad y cauce pequeño. Sin uso específico.

c. Área interserrana (A3): comprende el $80 \%$ del predio y se subdivide de acuerdo a los factores físicos asociados a grado de rocosidad, pendiente, exposición y la distribución de las comunidades naturales (pajonal, arbustal, roquedal, "flechillar" y roquedales con líquenes). El uso es espontáneo de tipo recreativo con actividades de senderismo.

Figura № 3: Delimitación de unidades ambientales síntesis Fuente: Elaboración personal en base a google earth

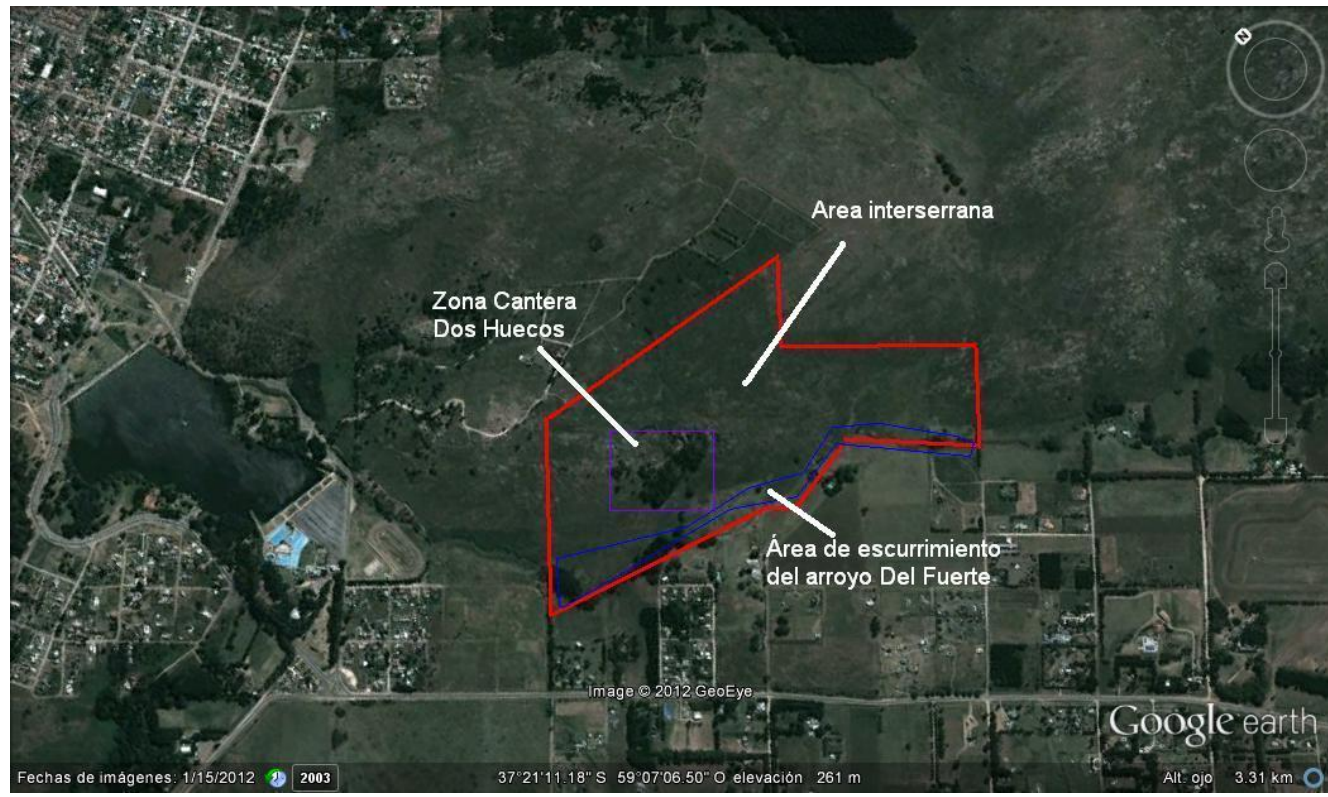




\section{Matriz de síntesis territorial Fuente: elaboración personal}

\begin{tabular}{lllllll}
\hline Unidades & Ecología & $\begin{array}{l}\text { Producción/ } \\
\text { uso }\end{array}$ & Protección & Paisaje & Patrimonio & Conservación \\
Área 1 & 1 & 3 & 3 & 2 & 3 & 2 \\
Área 2 & 2 & 1 & 3 & 1 & 2 & 1 \\
Área 3 & 3 & 2 & 2 & 3 & $2-3$ & 3 \\
\hline
\end{tabular}

Del análisis de la matriz se observa que:

- El Área 1 posee un valor elevado en relación a la presencia de elementos asociados al patrimonio minero y la historia de la minería artesanal en Tandil, por ende está vinculado a la necesidad de protección y conservación, aunque presenta un uso elevado, sobre todo para actividades deportivas de escalada y rapel en los frentes de cantera.

- $\quad$ El Área 2, coincidente con el valle del arroyo, presenta mayor vulnerabilidad, con un valor ecológico medio, sin embargo, éste se potencia combinado con otros dentro del predio y un uso actual bajo.

- El Área 3, la de mayor tamaño, posee un elevado valor en relación a su patrimonio natural, a la necesidad de protección del mismo y sobre todo por su valor paisajístico intrínseco, por las vistas que se obtienen en diferentes puntos panorámicos.

La síntesis indica que en el patrimonio natural y cultural combinado y la protección, están algunos elementos claves a considerar en relación a los usos.

Actividades. Dentro de las actividades propuestas, se establecen aquellas de uso actual, las que se enmarcan dentro del uso público recreativo tal como lo establece la ordenanza de donación y aquellas que se asocian con la reapropiación del patrimonio en dicho contexto.

a. Actividades recreativas de aventura: actividades de cuerda (rapel, escalada a.1); senderismo y treeking a.2; mountain bike a.3; cabalgatas a.4; cuatriciclos a.5, actividades de aventura combinadas "parque de aventura a.6.

b. Miradores y puntos panorámicos o de observación de flora y fauna.

c. Actividades de interpretación y senderismo asociado

d. Turismo educativo: vida en la naturaleza, orientación.

e. Actividades recreativas contemplativas y convencionales.

f. Actividades en torno al patrimonio minero-geológico.

Matriz de aptitud

Fuente: Elaboración personal

\begin{tabular}{llllllllllll}
\hline Unidad & a.1 & a.2 & a.3 & a.4 & a.5 & a.6 & B & C & d & e & $\mathbf{f}$ \\
Área 1 & 3 & 2 & 1 & 1 & 0 & 3 & 2 & 3 & 3 & 3 & 3 \\
Área 2 & 1 & 3 & 1 & 1 & 0 & 1 & 1 & 3 & 3 & 2 & 0 \\
Área 3 & 0 & 3 & 2 & 2 & 1 & 2 & 3 & 3 & 3 & 3 & 1 \\
\hline
\end{tabular}

Del análisis de la matriz de aptitud se desprende que;

- El A1 presenta sus mayores valores para actividades de cuerda y de aventura en general, así como para actividades de interpretación y de "turismo educativo" vinculadas a su representatividad dentro del patrimonio geológico y minero. Presenta restricciones para actividades de aventura de alto impacto (cuatriciclos y en menor medida cabalgatas y mountain bike).

- $\quad$ El A2 es la que presenta mayores limitaciones por su atractividad, aunque con valores altos respecto a actividades de senderismo y de interpretación ambiental, por su singularidad dentro del predio, único curso de agua existente. Sin embargo no deja de ser un área complementaria y perimetral.

- El A3 presenta mayor diversidad de escenarios y de alternativas para actividades de senderismo, con un fuerte vínculo con la interpretación patrimonial de valorización del pastizal pampeano incluso dentro del "turismo educativo". La homogeneidad interna requiere la identificación de sectores puntuales para usos específicos. Posee el mayor potencial para el desarrollo de propuestas de puntos panorámicos y cuencas visuales hacia el interior del predio y también relacionadas al espacio urbano y rural próximo. 
Matriz de impactos

Fuente: Elaboración personal

\begin{tabular}{llllllllllll}
\hline Unidad & $\mathbf{a . 1}$ & $\mathbf{a . 2}$ & $\mathbf{a . 3}$ & $\mathbf{a . 4}$ & $\mathbf{a . 5}$ & $\mathbf{a . 6}$ & $\mathbf{B}$ & $\mathbf{C}$ & $\mathbf{d}$ & $\mathbf{E}$ & $\mathbf{F}$ \\
Área 1 & 1 & 1 & 2 & 3 & 3 & 2 & 1 & 1 & 1 & $2^{\mathrm{EI}}$ & 1 \\
Área 2 & 0 & 2 & 3 & 3 & 3 & 0 & $1 \mathrm{El}$ & 1 & $1^{\mathrm{El}}$ & 2 & 0 \\
Área 3 & 0 & $1 \mathrm{El}$ & 2 & 2 & 3 & 2 & 2 & $1^{\mathrm{El}}$ & $1^{\mathrm{EI}}$ & $1^{\mathrm{EI}}$ & $1^{\mathrm{EI}}$ \\
\hline
\end{tabular}

En la matriz de impactos, se observa que para las actividades sugeridas en la reutilización del patrimonio (paisaje, pastizal y actividad minera artesanal), se debe establecer la menor cantidad de infraestructura y equipamiento posibles, por lo que se sugiere implementar evaluaciones de impacto ambiental (El). Reducir la carga de estos elementos y de la concentración sobre todo en el área 1, tanto de actividades como de visitantes, propiciando que el área 3 mantenga una dinámica de circulación, sin intervenciones en el terreno con impacto visual excesivo, con excepciones. Se observa la necesidad de revisar las actividades de aventura, evitando el uso de cuatriciclos, cabalgatas y bicicletas en menor medida y estableciendo controles, sobre todo porque estas últimas, demandan senderos particulares y pueden provocar procesos de carcavamiento y erosión, además necesitan espacios propios, no pudiendo convivir con las actividades recreativas contemplativas, pedestres, educativas o de recreación y aventura en general.

\section{Matriz de capacidad de Acogida \\ Fuente: Elaboración personal}

\begin{tabular}{llllllllllll}
\hline Unidad & $\mathbf{a . 1}$ & $\mathbf{a . 2}$ & $\mathbf{a . 3}$ & $\mathbf{a . 4}$ & $\mathbf{a . 5}$ & $\mathbf{a} .6$ & $\mathbf{B}$ & $\mathbf{C}$ & $\mathbf{d}$ & $\mathbf{E}$ & $\mathbf{F}$ \\
Área 1 & 3 & $3^{\mathrm{EI}}$ & 2 & 2 & 1 & 2 & 2 & 3 & 3 & 2 & 4 \\
Área 2 & 0 & 2 & $2 / 1$ & $2 / 1$ & 1 & 0 & 2 & 3 & $3^{\mathrm{EI}}$ & 2 & 0 \\
Área 3 & 0 & $3^{\mathrm{EI}}$ & 2 & 2 & 1 & 2 & 2 & $3^{\mathrm{EI}}$ & $3^{\mathrm{El}}$ & 2 & $3^{\mathrm{EI}}$ \\
\hline
\end{tabular}

La matriz de capacidad de acogida establece una vocación del patrimonio territorial para las actividades de turismo aventura, educativo, de interpretación y valorización del patrimonio, esto teniendo en cuenta el componente relacional: es decir, la integración funcional con un entorno recreativo de uso público muy intenso (Lago del Fuerte, Cerro de la Cruz, Paseo Cervantes, Paseo del Bicentenario); la integración física en un contexto de paisaje protegido y de corredores de vegetación contemplada en la Ley Provincial 14.126/10; y la social a partir del aumento regulado del uso público y del control del mismo por la Asociación de Guías y el Municipio de Tandil, el cual por ser el responsable legal de este espacio debe ser quien articule las iniciativas de uso del espacio.

\section{CONCLUSIONES}

Considerando los resultados obtenidos se puede indicar que las actividades compatibles que no tienen limitaciones requieren de una evaluación particular, ya que estamos frente a un área protegida de uso público. Es necesario evaluar la capacidad de carga de visitantes e incluso de infraestructura, ya que para el desarrollo de muchas actividades se requiere de equipamientos que pueden afectar negativamente el ambiente, por ejemplo, es el caso de las estructuras relacionadas a parques de aventura o simplemente las instalaciones necesarias para la práctica de actividades de cuerda.

En relación al patrimonio minero y geológico se puede mencionar que los frentes de canteras son valorados actualmente por diversos actores (deportistas, escuelas, tours operadores, residentes, etc.) como escenarios para realizar actividades de aventura, básicamente actividades de rapel y escalada, pero con escasa relación a la historia que existe detrás de esa transformación territorial producida por más de un siglo de trabajo minero.

Por esto es necesario complementar esa función actual, relacionada al turismo aventura, con la propia identidad de esos elementos en su relación histórica y territorial, en el marco de un programa elaborado especialmente desde lo que se conoce como turismo educativo y también haciendo uso de las técnicas aportadas desde la interpretación ambiental, las cuales pueden presentar de una forma amena y atractiva el patrimonio territorial aportando a la resignificación del mismo.

Pero el desarrollo de estas actividades debe estar conectadas también con otros elementos del territorio, donde se están realizando actualmente procesos de revalorización y reapropiación del patrimonio, por ejemplo, como son los talleres de picapedreros propiciados desde el Municipio o a otras áreas mineras cercanas donde puedan realizarse otro tipo de actividades vinculadas al patrimonio natural y/o cultural.

En definitiva, en términos potenciales es viable la reutilización del Parque Antonio y Juana Cinque como un espacio que puede permitir la reapropiación del patrimonio local serrano por parte de la comunidad e incluso para abrirlo a partir de su resignificación, considerando los cambios sociales y económicos. A su vez la aplicación de la metodología nos pone frente a otra hipótesis de que es necesario pensar el territorio en lo particular y lo general. Es decir, es necesario, en la caracterización de este sistema complejo, entender que la resignificación de un lugar y su apropiación es interdependiente y a su vez interdefinible por la trama de representaciones y 
acciones del territorio en su conjunto. La capacidad de una porción del territorio requiere de un juego de escalas permanentes, integrando así el área al contexto espacial y temporal que le otorga un nuevo significado. El conjunto (las sierras, la ciudad y el sistema turístico en su totalidad) son contextos o entornos que influyen y son influidos en la capacidad de acogida de un sitio y su relación pulsa fuerzas permanentes, donde se otorga significado y se construye la apropiación del territorio de lo cotidiano.

\section{BIBLIOGRAFÍA}

Adad, L. D. (2010) "Patrimonio, Identidad y Desarrollo: Breve ensayo sobre los procesos de Valoración, Apropiación y Usos del Patrimonio Cultural”. Newsletter № 15. Departamento de Antropología Social. FACSO, Tandil.

De Sola Morales, I. (1996). "Terrain Vague" IX Congreso de la UIA. Arquitectura de Ciudades. Presente y futuro, Barcelona. Disponible en http://bibliotecavirtual.clacso.org.ar/ar/libros/reggen/pp12.pdf

Fernández; G., Valenzuela; S., Ricci; S., Castronovo, R. y Ramos, A. (2014) "Capacidad de acogida del territorio para usos recreativo-educativos en una cantera inactiva de Tandil, Buenos Aires, Argentina”. Revista Estudios Ambientales. Vol 2, N 1, pp. 59-83.

Galacho Jiménez, F. B. y J. A. Arrebola Castaño (2008). "El modelo de evaluación de la capacidad de acogida del territorio. Aspectos conceptuales y técnicas relacionadas" Revista Baética. № 308, Universidad de Málaga.

Gómez Orea, D. (1992) Evaluación de Impacto Ambiental. Ed. Agrícola española, Madrid, España.

González Méndez, M. (2000). "Memoria Historia y Patrimonio. Hacia una concepción social del patrimonio. Trabajos de Prehistoria 57 № 2-9-22.

Guerrero Valdebenito, R. M. (2005) "Identidades territoriales y Patrimonio Cultural: La apropiación del patrimonio mundial en los espacios urbanos locales”. F@ro №2 Facultad de Ciencias Políticas y Sociales. UNAM. Valparaiso. Año 1, №2.

IAPH (coord.): Bases para una Carta sobre Patrimonio y Desarrollo en Andalucía. Sevilla: Junta de Andalucía, Consejería de Medio Ambiente, D.L. 1996

Leff, E. (2005) La geopolítica de la biodiversidad y el desarrollo sustentable: economización del mundo, racionalidad ambiental y reapropiación social de la naturaleza. en: semináro internacional reg en: alternativas globalização (8 al 13 de octubre de 2005, Hotel Gloria, Gio de Janeiro, Brasil). Rio de Janeiro, Brasil UNESCO, Organización de las Naciones Unidas para la Educación, la Ciencia y la Cultura.

Leff. E. (2007). ¿de quién es la naturaleza? Sobre la reapropiación social de los recursos naturales. www2.ine.gob.mx/publicaciones/gacetas/231/eleff.html

Lowenthal, D. (1998) El pasado es un país extraño. Akal, Madrid

Martín-Barbero, J. (1999) "Patrimonio: el futuro que habita la memoria". Somos Patrimonio: 91 Experiencias de Apropiación Social del Patrimonio Cultural y Natural. Convenio Andrés Bello, Bogotá.

Martínez Yáñez, C. (2008) "Patrimonialización del territorio y territorialización del patrimonio". Cuad. Art. Gr., 39, 251-266.

Molina Valencia, N. (2013) "Discusiones acerca de la resignificación". Revista MEC-EDUPAZ, Número III Septiembre-Marzo. Universidad Nacional Autónoma de México.

Teruggi, M., y J. Kilmurray. (1980) "Sierras septentrionales de la provincia de Buenos Aires". Geología Regional Argentina. Vol II, pp. 919-985, Córdoba, Argentina. 